\title{
Hypo, Hyper, or Combo: new paradigm for treatment of acute myeloid leukemia in older people
}

\author{
Gunnar Juliusson, ${ }^{1}$ Martin Höglund ${ }^{2}$ and Sören Lehmann ${ }^{2,3}$
}

'Department of Hematology, Skåne University Hospital, Lund, and Department of Hematology, Stem Cell Center, Department of Laboratory Medicine, Lund University, Lund; ${ }^{2}$ Department of Medical Sciences, Uppsala University, Uppsala and ${ }^{3}$ Department of Medicine, Karolinska Institute, Stockholm, Sweden

E-mail: GUNNAR JULIUSSON - gunnar.juliusson@med.lu.se

doi:10.3324/haematol.2019.238857

A cute myeloid leukemia (AML) ${ }^{1}$ is a serious disease. Using the combination of cytarabine and daunorubicin developed during the 1970s, ${ }^{2}$ with or without subsequent allogeneic stem cell transplantation (alloSCT), ${ }^{3}$ we now cure more than half of all patients with de novo AML up to the age of 60 years. ${ }^{4}$ But the outcome of older patients, that constitute the vast majority, remains dismal. The median age of AML is over 70 years, ${ }^{5}$ and the 3 -year overall survival (OS) of patients aged $70-84$ years is still less than $20 \%$ with intensive chemotherapy, and much worse in AML arising after myelodysplastic syndrome (MDS) or myeloproliferative neoplasia (MPN), ${ }^{5-7}$ or with palliative treatment only. ${ }^{5}$ However, new treatment options are finally emerging. ${ }^{1}$

In this issue of Haematologica, Talati et al. present a large $(\mathrm{n}=980)$, retrospective, single-center study on AML patients aged 70 years and older diagnosed between 1995 and 2016. Intensive combination chemotherapy was given to $37 \%$, hypomethylating agents (HMA) to $26 \%$, and other low-intensity or palliative treatment to $37 \%$. It is well established that specific AML therapy provides better outcome than palliation only., However, in this study, better survival rates were observed with HMA treatment than with intensive therapy (median 14.4 months vs. 10.8 months; $P=0.004$ ).

The currently approved HMA, 5-azacitidine (Aza) and decitabine (Dec, 5-aza-2'-deoxycytidine) are analogs of the natural pyrimidine cytidine. Dec incorporates into DNA while Aza mainly incorporates into RNA, and to a lesser extent into DNA (Figure 1). DNA incorporation causes hypomethylation by irreversible inhibition of DNA methyl transferases (DNMT) and upregulation of tumor suppressor genes, but it also leads to induction of DNA damage response. ${ }^{10}$ The cytotoxic effects are more evident at higher doses while hypomethylation seems to dominate at lower drug concentrations. ${ }^{11}$ Incorporation into RNA leads to inhibition of transfer RNA methylation, ultimately resulting in impaired messenger RNA transcription and protein synthesis. ${ }^{10,12}$ In addition, HMA have been shown to affect the immune system in various ways, such as upregulation of tumor antigens and induction of viral defense systems through upregulation of endogenous retroviruses. ${ }^{10,13}$ Intriguingly, it is still unclear what mechanisms are mostly responsible for the clinical effects of HMA in AML.

Aza and Dec were both synthesized in 1964, and biological activity was shown in mice. Clinical trials started in the 1970s. ${ }^{14}$ Initially, the maximal tolerated doses of Dec (1.5-2.5 g/m²/course) were tested with clinical activity but resulted in prolonged cytopenia. ${ }^{15}$ Subsequently de-escalated doses to the currently recommended 100 $150 \mathrm{mg} / \mathrm{m}^{2} /$ course of Dec or $525 \mathrm{mg} / \mathrm{m}^{2} /$ course of Aza became used for treatment of MDS, for which the US Food and Drug Administration (FDA) in 2004 approved Aza and in 2006 approved Dec. At the time of these studies, the diagnosis entity MDS included patients with bone marrow blasts up to $30 \%$. When MDS with 20 $30 \%$ blasts were later reclassified as $\mathrm{AML},{ }^{16} \mathrm{HMA}$ was approved also for AML with low blast counts. ${ }^{17}$ Studies in AML with $>30 \%$ blasts then started, ${ }^{18}$ leading to approval in 2015 of Aza for all patients with AML not eligible for intensive treatment.

The present study by Talati et al. ${ }^{8}$ confirms the activity of HMA in older patients and is the first to show improved survival with HMA in AML patients aged 70 years and older as compared to intensive treatment, in contrast to other studies ${ }^{19-22}$ (Table 1 and Figure 1). However, this discrepancy might be of limited importance, for two reasons. Firstly, in clinical practice, we should try to find the optimal therapy for each individual patient, rather than 'one treatment fits all'. Secondly, rapidly emerging therapeutic options may well replace

Table 1. Survival of older acute myeloid leukemia patients with hypomethylating or intensive treatment.

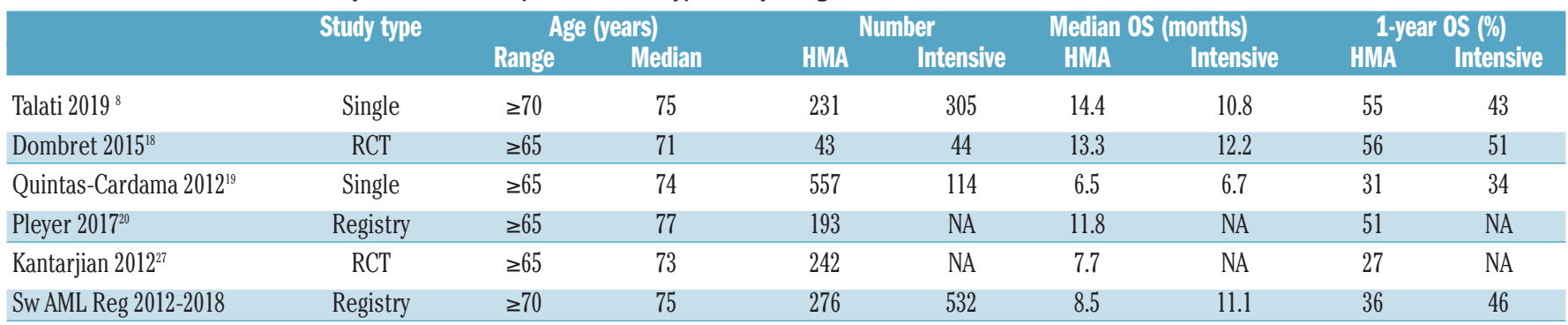

OS: overall survival; HMA: hypomethylating agents; NA: not assessed; Single: single-center study; RCT: multicenter randomized clinical study; Sw AML Reg: Swedish AML Registry, including patients diagnosed from 2012, with survival data updated in September 2019. 
A

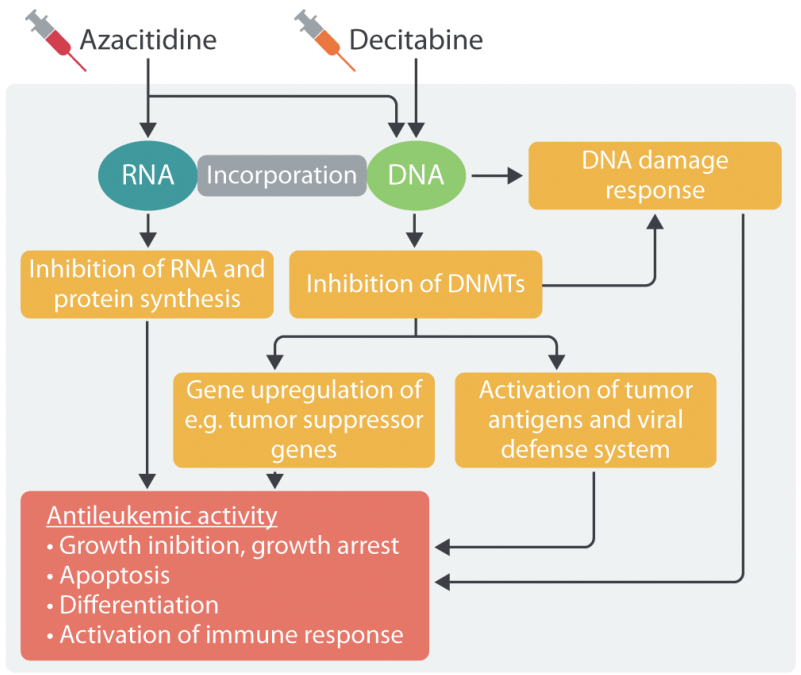

C

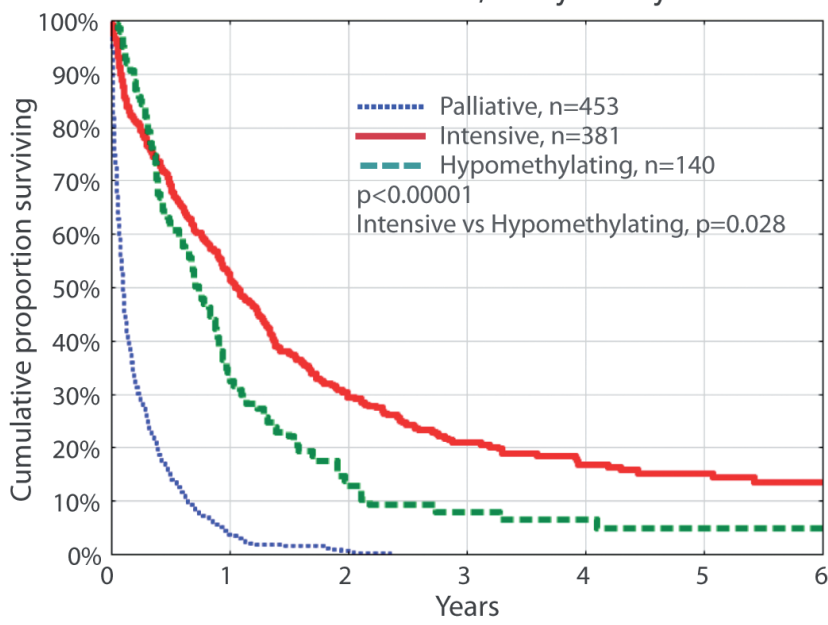

B

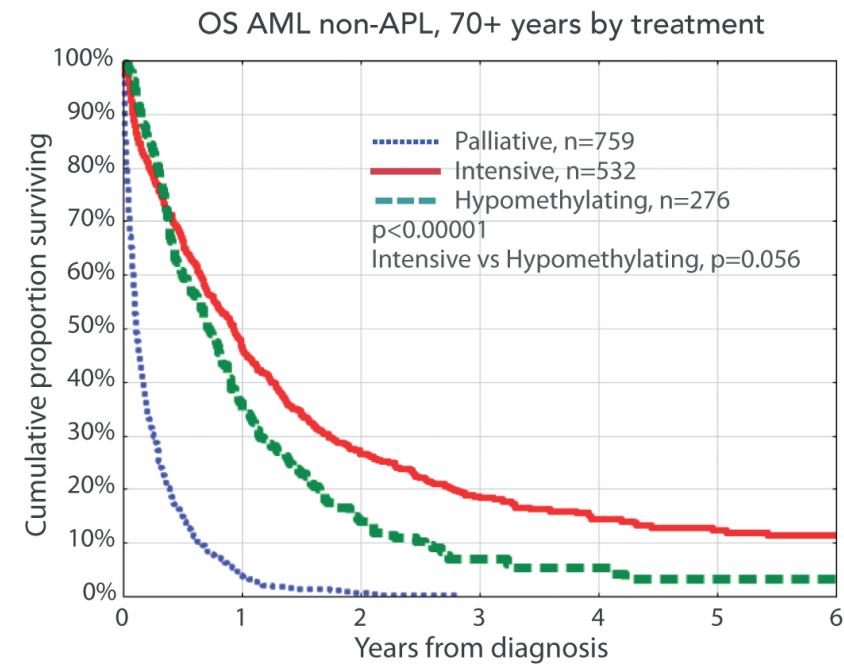

D

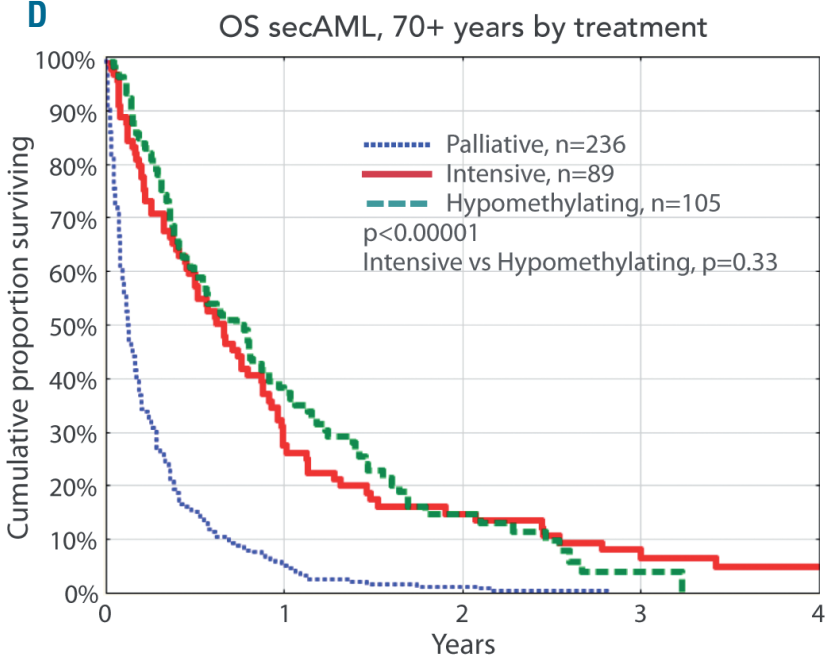

Figure 1. Mechanisms of action of hypomethylating agents, and overall survival of Swedish patients 70 years and older by treatment. (A) Mechanism of action for treatment with hypomethylating agents (HMA). DNA methyl transferases (DNMT). (B) Overall survival (OS) according to therapy. Acute promyelocytic leukemia is excluded (non-APL). All patients 70 years and older. HMA versus intensive treatment; $P=0.056$. (C) de novo acute myeloid leukemia (AML). HMA versus intensive treatment; $P=0.028$. (D) AML secondary to previous myelodysplastic syndromes (MDS), myeloproliferative neoplasia (MPN) or chemo-radiotherapy (secAML). HMA versus intensive treatment; $P=0.33$.

monotherapy in the near future.

Intensive AML treatment is toxic and requires massive supportive care and long-term hospitalization. Older patients with comorbidity may not tolerate this, even though half of the selected older patients achieve complete remission from intensive treatment, ${ }^{5}$ and mostly so within one month. Response to HMA is less frequent and often takes much longer to achieve, but is usually well manageable in the outpatient department. The role of intensive consolidation and maintenance has still not been determined, and HMA may serve as maintenance also after intensive chemotherapy. ${ }^{23}$ Importantly, subsets of AML respond well to intensive treatment, whereas patients with secondary AML and/or complex or MDSlike genetics do poorly with chemotherapy ${ }^{5,7}$ (Figure 1), and such patients are better off with HMA. Responding patients may be eligible for alloSCT as a curative approach, whether response was achieved from intensive treatment or from HMA.

Clinical and academic studies always introduce patient selection, and the interpretation of retrospective non-randomized trials should include consideration of potential differences in the actual management of different patient subsets. This problem can be overcome in part by assessing patients' characteristics, prognostic factors and propensity score matching. The Talati et al. study includes a large number of patients diagnosed since 1995, i.e. some of them were treated as part of the early development of HMA. ${ }^{8}$ However, results were similar when comparing outcome for patients diagnosed before and after 2005. What is striking is that half the Moffitt Cancer Center patients had prior MDS (some also had prior HMA treatment), as compared to $18 \%$ in the AML-001 study, ${ }_{10}^{18} 25 \%$ in the Austrian registry study, ${ }^{20} 12 \%$ in the Danish popu- 
lation-based study including all ages, ${ }^{6}$ and $28 \%$ of patients aged $\geq 70$ years given AML-specific treatment according to the Swedish AML Registry.,5 Still, the Talati et al. study had the best reported median survival with HMA and is the only study so far to show better survival with HMA than with intensive treatment (Table 1). ${ }^{8}$

The therapeutic options for AML in older people are now rapidly expanding, including oral targeted drugs with low toxicity, such as kinase inhibitors with activity in AML with FLT3-mutations, oral inhibitors of IDHmutations, and many more. ${ }^{1}$ These drugs have been shown to be active as monotherapy, and some are already approved by the FDA. However, both theory and practice indicate synergistic effects of combining drugs with different modes of action. Clinical studies are, therefore, rapidly moving towards regimes with HMA used as backbone therapy, comparing monotherapy with add-on of further drugs, of which there are several. Expectations are high from the combination of HMA with the BCL2inhibitor venetoclax, that has resulted in very high complete remission (CR) rates $(67 \% \mathrm{CR}+\mathrm{CR}$ with incomplete blood count recovery) and a median overall survival of 17.5 months when used as primary treatment of older AML patients (median 74 years), ${ }^{24}$ although real-world response rates were somewhat lower. ${ }^{25}$ (The outcome of the phase III study with Aza + venetoclax/placebo may be presented in 2020.) Furthermore, an oral analog of Aza (CC-486) has recently been reported to be effective to maintain remission from AML. ${ }^{26}$ We thus expect numerous studies evaluating HMA-based new combinations, and all-oral limited-toxicity treatments are within sight.

In summary, traditional combination chemotherapy with or without the addition of targeted therapies is likely to keep its role for years to come for many patients without severe comorbidity up to the age of around 75 years. However, patients with secondary AML and/or high-risk genetics should now already be offered less toxic HMA-based therapies, preferably as part of one of the many ongoing clinical trials, in order to expand our clinical armamentarium as quickly as possible.

\section{References}

1. Döhner H, Estey E, Grimwade D, et al. Diagnosis and management of AML in adults: 2017 ELN recommendations from an international expert panel. Blood. 2017;129(4):424-447.

2. Yates JW, Wallace HJ Jr, Ellison RR, Holland JF. Cytosine arabinoside (NSC-63878) and daunorubicin (nsc-83142) therapy in nonlymphocytic leukemia. Cancer Chemother Rep. 1973;57(4):485-488.

3. Cornelissen JJ, Gratwohl A, Schlenk RF, et al. The European LeukemiaNet AML Working Party consensus statement on allogeneic HSCT for patients with AML in remission: an integrated-risk adapted approach. Nat Rev Clin Oncol. 2012;9(10):579-590.

4. Juliusson G, Hagberg O, Lazarevic VL, et al. Improved survival of men 50-75 years old with acute myeloid leukemia over a 20 -year period. Blood. 2019;134(18):1558-1561.

5. Juliusson G, Antunovic P, Derolf A, et al. Age and acute myeloid leukemia: real world data on decision to treat and outcomes from the Swedish Acute Leukemia Registry. Blood. 2009;113(18):41794187.

6. Granfeldt Östgård LS, Medeiros BC, Sengelöv H, et al. Epidemiology and clinical significance of secondary and therapy-related acute myeloid leukemia: a national population-based cohort study. J Clin Oncol. 2015;33(31):3641-3649.
7. Hulegårdh E, Nilsson C, Lazarevic V, et al. Characterization and prognostic features of secondary acute myeloid leukemia in a population-based setting: a report from the Swedish Acute Leukemia Registry. Am J Hematol. 2015;90(3):208-214.

8. Talati C, Dhulipala VC, Extermann M, et al. Comparisons of commonly used frontline regimens on survival outcomes in patients age 70 years and older with acute myeloid leukemia. Haematologica. 2020;105(2):398-406

9. Oran B, Weisdorf DJ. Survival for older patients with acute myeloid leukemia: a population-based study. Haematologica. 2012;97(12): 1916-1924.

10. Diesch J, Zwick A, Garz AK, Palau A, Buschbeck M, Götze KS. A clinical-molecular update on azanucleoside-based therapy for the treatment of hematologic cancers. Clin Epigenetics. 2016;8:71.

11. Saiki JH, Bodey GP, Hewlett JS, et al. Effect of schedule on activity and toxicity of 5-azacytidine in acute leukemia: a SWOG study. Cancer. 1981;47(7):1739-1742.

12. Bohl SR, Bullinger L, Rücker FG. Epigenetic therapy: azacytidine and decitabine in acute myeloid leukemia. Expert Rev Hematol. 2018;11(5):361-371.

13. Chiappinelli KB, Strissel PL, Desrichard A, et al. Inhibiting DNA Methylation Causes an Interferon Response in Cancer via dsRNA Including Endogenous Retroviruses. Cell. 2015;162(5):974-986.

14. Von Hoff DD, Slavik M, Muggia FM. 5-azacytidine. A new anticancer drug with effectiveness in acute myelogeneous leukemia. Ann Intern Med. 1976;85(2):237-245.

15. Jabbour E, Issa JP, Garcia-Manero G, Kantarjian H. Evolution of Decitabine. Development Accomplishments, Ongoing Investigations, and Future Strategies. Cancer. 2008;112(11):23412351.

16. Vardiman JW, Harris NL, Brunning RD. The World Health Organization (WHO) classification of the myeloid neoplasms. Blood. 2002;100(7):2292-2302.

17. Fenaux P, Mufti GJ, Hellström-Lindberg E, et al. Azacytidine prolongs overall survival compared with conventional care regimens in elderly patients with low bone marrow blast count acute myeloid leukemia. J Clin Oncol. 2010;28(4):562-569.

18. Dombret $\mathrm{H}$, Seymour JF, Butrym A, et al. International phase 3 study of azacytidine vs conventional care regimens in older patients with newly diagnosed AML with $>30 \%$ blasts. Blood. 2015;126(3):291299.

19. Quintas-Cardama A, Ravandi F, Liu-Dumlao T, et al. Epigenetic therapy is associated with similar survival compared with intensive chemotherapy in older patients with newly diagnosed acute myeloid leukemia. Blood. 2012;120(24):4840-4845

20. Pleyer L, Döhner H, Dombret H, et al. Azacitidine for front-line therapy of patients with AML: reproducible efficacy established by direct comparison of international phase 3 trial data with registry data from the Austrian Azacytidine Registry of the AGMT study group. Int J Mol Sci. 2017;18(2):E415.

21. Schuh AC, Döhner H, Pleyer L, Seymour JF, Fenaux P, Dombret H. Azacitidine in adult patients with acute myeloid leukemia. Crit Rev Oncol Hematol. 2017:116:159-177.

22. Österros A, Eriksson A, Antunovic P, et al. Real-world data on treatment patterns and outcomes of hypomethylating therapy in newly diagnosed acute myeloid leukemia $>60$ years. Br J Haematol. 2020; doi: 10.1111/bjh.16410.

23. Huls G, Chitu DA, Havelange V, et al. Azacitidine maintenance after intensive chemotherapy improves DFS in older AML patients. Blood. 2019;133(13):1457-1464.

24. DiNardo CD, Pratz K, Pullarkat V, et al. Venetoclax combined with decitabine or azacytidine in treatment-naïve, elderly patients with acute myeloid leukemia. Blood. 2019;133(1):7-17.

25. Winters AC, Gutman JA, Purev E, et al. Real-world experience of venetoclax with azacitidine for untreated patients with acute myeloid leukemia. Blood Adv. 2019;3(20):2911-2919.

26. Wei AH, Döhner H, Pocock C, et al. The QUAZAR AML-001 Maintenance Trial: Results of a Phase III International, Randomized Double-Blind, Placebo-Controlled Study of CC-486 (Oral Formulation of Azacitidine) in Patients with Acute Myeloid Leukemia (AML) in First Remission. Blood. 2019;134:Supplement_2: LBA-3.

27. Kantarjian HM, Thomas XG, Dmoszynska A, et al. Multicenter, randomized, open-label, phase III trial of decitabine versus patient choice, with physician advice, of either supportive care or low-dose cytarabine for the treatment of older patients with newly diagnosed acute myeloid leukemia. J Clin Oncol. 2012;30(21):2670-2677. 